\title{
Innovación aplicada al desarrollo del liderazgo lasaliano en Brasil: fundamentos para la excelencia en la gestión
}

\author{
RENALDO VIEIRA DE SOUZA* \\ HILDEGARD SUSANA JUNG ** \\ PAULO FOSSATTI***
}

Artículo de investigación sobre innovación y liderazgo en una universidad lasaliana en Brasil.

Recibido: 30 de abril de 2020 - Evaluado: 10 de agosto de 2020 - Aceptado: 25 de septiembre de 2020

Citar como: Vieira de Souza, R., Jung, H. y Fossatti, P. (2021). Innovación aplicada al desarrollo del liderazgo lasaliano en Brasil: fundamentos para la excelencia en la gestión. Hallazgos, 18(35), 105-128. DoI: https://doi.org/10.15332/2422409X.5757

* Doctor en Educación. Investigador y docente en la Universidad La Salle, Brasil. Integrante del grupo de investigación Gestión Educacional en los Diferentes Contextos.

Correo electrónico: renaldo.souza@unilasalle.edu.br

ORCID: https://orcid.org/0000-0003-2282-7394

** Doctora en Educación. Docente en la Universidad La Salle, Brasil. Integrante del grupo de investigación Gestión Educacional en los Diferentes Contextos.

Correo electrónico: hildegard.jung@gmail.com

ORCID: https://orcid.org/0000-0001-5871-3060

*** Doctor en Educación. Rector y docente del programa de Posgrado en Educación de la Universidad La Salle, Brasil. Líder del Grupo de Investigación Gestión Educacional en los Diferentes Contextos.

Correo electrónico: paulo.fossatti@unilasalle.edu.br ORCID: https://orcid.org/0000-0002-9767-5674 


\section{Resumen}

Este artículo trata sobre la aplicación de los fundamentos de la excelencia en gestión al desarrollo de liderazgos de la Universidad La Salle (Unilasalle), Brasil. Liderar es un ejercicio que exige autoconocimiento y constante perfeccionamiento de habilidades y competencias. Cuando se trata de innovación aplicada al liderazgo, las buenas prácticas y las nuevas metodologías de apoyo a la gestión son esenciales para auxiliar la práctica del liderazgo. Con base en esto, el objetivo de este artículo es presentar el proceso de desarrollo de liderazgos organizacionales en la Unilasalle. La metodología se basa en análisis documental, diario de campo y deposiciones de liderazgos y de la percepción de los equipos. Los resultados señalan: formación sistematizada y continuada de los líderes; elección estratégica de los participantes; gestión por competencias; autodesarrollo personalizado y ajuste de la práctica de desarrollo del liderazgo de la Unilasalle basado en sus principios y valores. Se concluye con la necesidad de formar personas con competencias técnicas, humanas y transversales, con enfoque en gestión innovadora, estratégica y por resultados. Las actitudes del liderazgo transformador pasan necesariamente por un minucioso plan de trabajo y organización, fundamentales para el éxito del ejercicio del liderazgo.

Palabras clave: Educación superior; Gestión de excelencia; Liderazgo innovador; Universidad La Salle. 


\title{
Innovation applied to the development of Lasallian leadership in Brazil: foundations for excellence in management
}

\begin{abstract}
This article addresses the application of the fundamentals of management excellence to leadership development at La Salle University (Unilasalle), Brazil. Leading is an exercise that requires selfknowledge and constant improvement of skills and competencies. When it comes to innovation applied to leadership, good practices and new management support methodologies are essential to help the practice of leadership. Based on this, the objective of this article is to present the process of organizational leadership development at Unilasalle. The methodology is based on documentary analysis, field diary and statements of leaderships and the perception of the teams. Results indicate: systematized and continuous training of leaders; strategic choice of participants; management by competencies; personalized self-development and adjustment of the leadership development practice of Unilasalle based on its principles and values. It concludes with the need to train people with technical, human and cross-cutting capabilities, with a focus on innovative, strategic and resultsbased management. The attitudes of transformational leadership necessarily go through a detailed work plan and organization, which are fundamental to the success of the exercise of leadership.

Palabras clave: Higher education; Management excellence; Innovative leadership; La Salle University.

\section{Inovação aplicada ao desenvolvimento da liderança lassalista no Brasil: fundamentos para a excelência na gestão}

Resumo

Este artigo trata da aplicação dos fundamentos da excelência em gestão ao desenvolvimento de lideranças da Universidad e La Salle (Unilasalle), Brasil. Liderar é um exercício que exige autoconhecimento e constante aperfeiçoamento de habilidades e competências. Quando se trata de inovação aplicada à liderança, as boas práticas e as novas metodologias de apoio à gestão são essenciais para auxiliar a prática da liderança. Com base nisso, o objetivo deste artigo é apresentar o processo de desenvolvimento de lideranças organizacionais na Unilasalle. A metodologia está baseada na análise documental, no diário de campo, nas exonerações de lideranças e na percepção das equipes. Os resultados indicam: formação sistematizada e continuada dos líderes; escolha estratégica dos participantes; gestão por competências; autodesenvolvimento personalizado e ajuste da prática de desenvolvimento da liderança da Unilasalle baseado em seus princípios e valores. Conclui-se com a necessidade de formar pessoas com competências técnicas, humanas e transversais, com foco na gestão inovadora, estratégica e por resultados. As atitudes da liderança transformadora passam necessariamente por um detalhado plano de trabalho e organização, fundamentais para o sucesso do exercício da liderança.

Palavras-chave: Ensino superior; Gestão de excelência; Liderança inovadora; Universidad La Salle. 


\section{Introducción}

El tema de la gestión educacional ha recibido especial atención en el escenario educativo brasileño, principalmente en los últimos 20 años, con la promulgación de la actual Ley de Directrices y Bases de la Educación Nacional (LDB), Ley 9394 del 20 de diciembre de 1996. La normativa presenta disposiciones sobre la gestión democrática en los ámbitos de la educación básica y superior de las instituciones públicas; garantiza la participación docente y discente, y establece la formación de consejos que integran la comunidad escolar y los cuerpos colegiados, en el caso de las universidades (Brasil, 1996). Con base en esta concepción, en las últimas dos décadas han proliferado estudios acerca de la gestión (Lück, 2000; Cardoso, 2001), la gestión democrática (Paro, 1997; Cury, 2002), el liderazgo (Lück, 2012) y la gobernanza (Fossatti, GangaContreras y Jung, 2017; Fossatti, Souza y Contreras, 2015), que han abarcado factores y escenarios imbricados en esos conceptos.

Ahora se da un paso más allá, principalmente en el ámbito de la enseñanza superior, es decir, además de estudios sobre gestión, gobernanza y liderazgo, son bienvenidas las investigaciones sobre los conceptos, principios y buenas prácticas de formación de liderazgos, a las que se añaden las que tratan los liderazgos innovadores. Sobre este tema versa el presente artículo: sobre los fundamentos de la excelencia en gestión en el desarrollo de liderazgos de la Universidad La Salle, Brasil (Unilasalle), una institución perteneciente a la Red La Salle de Enseñanza Superior del Sur de Brasil.

En ese contexto, el objetivo de este documento consiste en presentar el proceso de desarrollo de liderazgos organizacionales en la Unilasalle. Para ello, la metodología, de abordaje cualitativo - puesto que está cargada de elementos subjetivos (Gil, 2008) - , se basa en análisis documental, diario de campo y deposición de liderazgos. Para el análisis de los datos se siguió el enfoque de análisis de contenido de Bardin (2016), que refiere la necesidad de aglomerar un máximo posible de documentos para que sean analizados según cuatro reglas principales: 1) regla de la exhaustividad, que busca un máximo de material sin abandonar el análisis de elementos, debido a la dificultad de acceso, poco interés, entre otros; 2) regla de la representatividad, que consiste en que la muestra seleccionada deberá ser suficientemente representativa para el tema de la investigación; 3) regla de la homogeneidad, en la que el material seleccionado debe ser elegido por los mismos criterios — que en este caso han sido las categorías: liderazgo innovador, educación superior, Universidad La Salle_; 4) pertenencia, que se refiere al hecho de que los documentos elegidos se 
deben ajustar al objetivo de la investigación, lo que permite la articulación de nuevos conocimientos.

De acuerdo con Sarmento, Menegat y Ramírez (2015), liderar una institución con éxito implica alcanzar los patrones de calidad y excelencia que les exigen a las instituciones en la actualidad, ya sea por parte de los órganos gubernamentales y/o privados competentes, de la comunidad o de su público interno y/o externo. Para ello no se puede actuar de forma inexperta, es decir, no es "posible administrar con base en una conducta de 'ensayo y error' y/o pautada exclusivamente por experiencias previas” (p. 314, traducción propia).

Liderar, por tanto, exige autoconocimiento y perfeccionamiento de habilidades y competencias para desempeñar satisfactoriamente su papel, hoy más basado en estudios sobre buenas prácticas que en el sentido común. Y cuando se trata de liderazgo innovador (Etzkowitz y Zhou, 2017), las buenas prácticas y las nuevas metodologías de apoyo a la gestión se vuelven esenciales. En este contexto, el proceso de desarrollo de liderazgos organizacionales en la Unilasalle se convierte en el objeto de estudio que podrá servir al perfeccionamiento de investigaciones futuras.

De esa manera, este artículo se divide en los siguientes apartados: inicia con esta introducción; luego se presentan los referentes teóricos de la investigación, dentro de los cuales se discute el concepto, los estilos y las premisas del liderazgo, el concepto de innovación y el de liderazgo innovador; después se expone la metodología; posteriormente se realiza el análisis y la discusión de los resultados, y culmina con unas consideraciones finales.

\section{Fundamentación teórica}

De acuerdo con Lück (2000), la particularidad de un líder es caracterizarse como una persona emprendedora, que se compromete a mantener la motivación del equipo, con determinación y autocontrol, sin dejar de ser flexible. Para esta autora es fundamental que el líder conozca los principios de la educación y sus procesos, pues de ese conocimiento vendrá su autoridad, de modo que entienda el comportamiento humano y sea consciente de las motivaciones, los intereses y las competencias del perfil del grupo al que pertenece.

El liderazgo normalmente se compone de un proceso en el que se involucra una relación de influencia en doble sentido, orientada especialmente a la atención de objetivos mutuos, que forman parte de un grupo, de una institución o de la sociedad. 
Así, el liderazgo no requiere tan solo el puesto de líder, sino también el esfuerzo de cooperación por parte de las otras personas (Hollander, 1978). Este asunto del liderazgo tiene cierta complejidad, puesto que se desarrolla en medio de la interacción entre los seres humanos, lo cual, de una forma u otra, trae consigo percepciones y vivencias distintas (Fossatti, 2013).

\section{Concepto, estilos y premisas de liderazgo}

Con relación al liderazgo, Bergamini (1994) lo refiere como algo que atañe tanto a los que administran como a los administrados. Para él, el liderazgo trasciende el don de responsabilidad sobre una especie, con la atracción enigmática que ejercen algunos individuos sobre otros. Según el autor, el término se ha desasociado de la figura autoritaria del "jefe", y se ha ubicado cerca del concepto de líder, que se refiere a una persona de "éxito", puesto que los líderes tienen "seguidores” y no "subordinados”.

Respecto a los rasgos y estilos de liderazgo, Bryman (1992) explica que los conceptos teóricos relacionados con los rasgos de personalidad son más antiguos en cuanto al liderazgo. Esas teorías identifican al líder como aquel que posee rasgos específicos de personalidad, que lo difieren de las demás personas. En ese sentido, Da Silva et ál. (2019) consideran que el liderazgo es una competencia esencial en los gestores. Así, el líder presenta características de personalidad por medio de las cuales puede influenciar el comportamiento de los demás. Como premisa de esa teoría, los líderes poseen rasgos de personalidad que permiten indicar que el individuo ya nace líder. Bryman (1992) todavía recalca tres importantes aspectos relacionados con los rasgos sociales, aquellos asociados a la tarea, desde el punto de vista del comportamiento de los seguidores: 1) la influencia que ejerce el líder sobre los demás, lo que en alguna medida induce su comportamiento; 2) la concepción de este proceso de influencia como incorporado al contexto de un grupo; 3) la influencia ejercida por el líder sobre el comportamiento de los miembros de un grupo hacia los objetivos que pretenden alcanzar. Este corpus conceptual lo va perfeccionado el propio autor, incluyendo asimismo las cuestiones culturales y de identificación — con la institución y con el grupo en sí-.

De esta manera, es posible observar una identificación del concepto de liderazgo con la influencia: influencia en los objetivos, las estrategias, el comprometimiento, el consentimiento sobre determinados comportamientos, para el alcance de los objetivos del grupo y su identificación mutua (Villarroel y Bruna, 2017). De ese modo, el liderazgo está condicionado de forma directa no solo en las organizaciones, sino también en todos los grupos sociales, formales e informales que buscan, a través del 
trabajo en común, un objetivo o la realización de determinada función (Zanguebuche et ál., 2019). Otro elemento importante se refiere a la percepción de las personas con relación a los tipos de liderazgo, tales como el liderazgo transaccional, que conduce o motiva a los seguidores hacia las metas establecidas por medio de la aclaración de los roles de cada uno y de las exigencias de las tareas. Un buen liderazgo motiva el equipo, lo que involucra más fuertemente a los colaboradores con las metas institucionales (da Silva et ál., 2019).

La teoría de los estilos de liderazgo cree que las personas se pueden preparar para ejercer el rol de líder. Para ello deben estudiar y establecer formas de ejercitar el poder, basadas en las creencias básicas sobre el hombre y la naturaleza humana (França y Arellano, 2002). Según Lickert (citado por Bergamini, 1994), existen dos estilos de jefatura que conciben los extremos entre participación y sumisión. El primero es el liderazgo orientado a las personas, que considera un mayor envolvimiento de ellas. El segundo estilo se orienta hacia la producción, en el cual las personas tan solo adoptan las determinaciones del gestor, representadas por cuatro patrones: 1) relación con poca interacción, basada en el miedo y en la desconfianza; 2) existencia de alguna confianza en la relación, con objetivos establecidos por el gestor y algún control intermediario; 3) confianza parcial de los gestores hacia los subordinados, ya que algunas decisiones son tomadas por los subordinados, lo cual los recompensa y a su vez los hace sentir responsables del alcance de los objetivos de la organización; 4) confianza plena de los gestores hacia los subordinados, quienes también participan en las decisiones.

Así mismo, de acuerdo con Lickert (citado por Bergamini, 1994), las actitudes del liderazgo transformador pasan necesariamente por un minucioso plan de trabajo y organización, que será fundamental para el éxito. Con esto, el autor destaca la necesidad de identificar algunos elementos esenciales: 1) definir objetivos claros; 2) comunicar al equipo, dejando claro lo que se espera, para lo cual debe desarrollarlo enseñando y aprendiendo, y estar atento a sus señales; 3) priorizar acciones y promover espacios de conocimiento; 4) asumir el papel de líder, verificando sistemáticamente el cumplimiento de las acciones, con absoluto respeto hacia las personas y ajustando los intereses individuales a los de la organización; 5) gerenciar de forma participativa a través de equipos, buscando en la función de las personas una palanca para su compromiso, y así motivarlas mediante el vínculo y la inspiración; 6) tener un instrumento para la competitividad y el crecimiento, haciendo de la comunicación la clave del envolvimiento y de la acción; 7) tener la cultura como ancla para mantener el negocio. 


\section{El concepto de innovación y de liderazgo innovador}

Según Bresciani y Guidelli (2010), el término innovación se refiere al uso de la tecnología en la fabricación de productos o en el ofrecimiento de servicios, e incide asimismo en la gestión organizacional. Para Castilhos (1998), la innovación se define como el uso, la aplicación y la transformación del conocimiento técnico y científico en la producción y la comercialización, cuya finalidad es el lucro. Las transformaciones utilizadas para comerciar nuevos productos también caben dentro de lo que se denomina innovación. A su vez, la innovación organizacional trata de las alteraciones en la forma de gestionar y organizar la producción. Participar en un mercado internacionalmente competitivo significa ofrecer productos y servicios hechos de formas diferentes y puestos lado a lado para la completa la elección de los clientes (Castilhos, 1998).

Según Siqueira (1995), la fuente de riqueza de una organización no son los bienes materiales, sino la inteligencia creativa, el capital intelectual propio de la forma de crear e innovar de las personas. Para el autor, el líder innovador incentiva en las personas las condiciones para que puedan desarrollar sus habilidades y aplicarlas en la mejora de los resultados de su trabajo. Los líderes creativos desarrollan en sus colaboradores las aspiraciones y expectativas, lo cual los transforma en auténticos emprendedores, dedicados a la excelencia de sus tareas y funciones (Lizuka, 2019). El liderazgo innovador, según Fossatti, Güths y Jung (2019), presupone calidad y excelencia, así como buenas prácticas y nuevas metodologías de apoyo a la gestión.

Para Sherer y Carlomagno (2009), una cultura de innovación demanda liderazgos empeñados y comprometidos, puesto que el desafío de la innovación es hacer que todos los líderes sean facilitadores del flujo de ideas y del conocimiento, y transformadores de la organización en su totalidad, pues si no hay implicación de los liderazgos con estrategias de innovación, podrán surgir barreras y dificultades.

\section{Metodología}

La investigación de la que surge este artículo se caracteriza por ser un estudio de caso con análisis documental sobre el modelo de gestión de una institución de enseñanza superior (IES) del sur de Brasil, cuyo abordaje cuantitativo ofrece aportes para el enfoque cualitativo (Gil, 2008). Para Lüdke y André (1986), el estudio de caso puede ser del tipo simple y específico, o complejo y abstracto. Los abordajes de los autores señalan que el estudio de caso presupone la observación de tres fases: la inicial es la 
exploratoria; en la segunda se realiza la delimitación del estudio y la colecta de datos; y en la tercera, el análisis sistemático y la estructuración del informe.

Ahora bien, este artículo se desprende de un proyecto de investigación en marcha titulado "Gestión Universitaria: de la teoría a los modos de actuación en diferentes contextos del Consorcio de las Universidades Comunitarias Gauchas (Comung)", del programa de Posgrado en Educación de la Unilasalle, Canoas, Brasil. En este recorte se abordan los factores de gestión universitaria que se relacionan directamente con la formación del perfil de liderazgos, con vistas a la excelencia académica, ajustados a los principios institucionales de una institución comunitaria de enseñanza superior (ICES).

Para llegar a los resultados parciales del trabajo, se ha realizado un análisis documental que involucró: Plan de Desarrollo Institucional (PDI, 2019); Informe de Gestión del Programa Gaucho de la Calidad y Productividad (RG-PGQP, 2015), entre otros documentos.

El estudio se apoya en la propuesta de análisis de contenido, presentada por Bardin (2016), para examinar el contenido y las directrices expresadas en el PDI y otros documentos que se despliegan a partir de este. Según Bardin (2016), las distintas fases del análisis de contenido son: el preanálisis, la exploración del material, el tratamiento de los resultados y la inferencia y la interpretación de los resultados.

Teniendo en cuenta que esta investigación aborda el modelo de gestión de la IES, perteneciente a la Red La Salle de Enseñanza Superior del Sur de Brasil, los análisis y hallazgos hasta aquí relatados podrán ser extendidos a las demás instituciones de la Red, lo que hace posible una comparación y una estandarización del modelo de gestión de liderazgos innovadores, en una forma de trabajo en red de cooperación.

\section{Análisis y discusión de los resultados}

Los resultados de la indagación se componen de los siguientes elementos: 1) formación sistematizada y continua de los líderes, 2) elección estratégica de los participantes, 3) gestión por competencias, 4) autodesarrollo personalizado y 5) ajuste de la práctica de desarrollo del liderazgo de la Unilasalle, basado en sus principios y valores, como veremos a continuación. 


\section{Formación sistematizada y continua de los líderes}

La Universidad La Salle (Unilasalle) demuestra el ajuste de la práctica de desarrollo del liderazgo a sus principios y valores, según se describe en la tabla 1.

Tabla 1. Conceptos orientadores de la gestión

\begin{tabular}{|c|c|c|}
\hline $\begin{array}{l}\text { Valores/principios } \\
\text { organizacionales }\end{array}$ & $\begin{array}{c}\text { Conceptos orientadores de la gestión } \\
\text { (directrices) }\end{array}$ & Partes involucradas \\
\hline Visión & $\begin{array}{l}\text { Ser, en } 2025 \text {, una universidad reconocida } \\
\text { por la excelencia académica y por la } \\
\text { internacionalización. }\end{array}$ & $\begin{array}{l}\text { Colaboradores, } \\
\text { Rectoría/directores, } \\
\text { aliados, comunidad }\end{array}$ \\
\hline Misión & $\begin{array}{l}\text { Promover formación integral y continua de } \\
\text { la persona por medio de la enseñanza, de la } \\
\text { investigación y de la extensión de excelencia } \\
\text { para el desarrollo sostenible de la sociedad, } \\
\text { fundamentado en los principios y en la } \\
\text { tradición cristiano-lasaliana. }\end{array}$ & $\begin{array}{l}\text { Clientes, colaboradores, } \\
\text { Rectoría/directores, } \\
\text { aliados, comunidad }\end{array}$ \\
\hline Principios y valores & $\begin{array}{l}\text { 1. Inspiración y vivencia cristiano-lasaliana } \\
\text { 2. Identidad católica y comunitaria } \\
\text { 3. Calidad y excelencia } \\
\text { 4. Valoración de las personas } \\
\text { 5. Colaboración y cooperación } \\
\text { 6. Ética } \\
\text { 7. Educación continua } \\
\text { 8. Desarrollo sostenible } \\
\text { 9. Espíritu emprendedor e Innovación } \\
\text { 10. Trabajo en red } \\
\text { 11. Internacionalización }\end{array}$ & $\begin{array}{l}\text { Clientes, colaboradores, } \\
\text { proveedores, Rectoría/ } \\
\text { directores, aliados, } \\
\text { comunidad }\end{array}$ \\
\hline $\begin{array}{l}\text { Negocio de la } \\
\text { Unilasalle }\end{array}$ & $\begin{array}{l}\text { Educación superior, cursos de pregrado, } \\
\text { posgrado, investigación y extensión, } \\
\text { proyectos y acciones sociales. }\end{array}$ & $\begin{array}{l}\text { Colaboradores, clientes, } \\
\text { aliados, proveedores, } \\
\text { comunidad }\end{array}$ \\
\hline
\end{tabular}

Fuente: Unilasalle $(2015 ; 2019)$.

La figura 1, a continuación, presenta la estructura organizacional de la Unilasalle. 
Figura 1. Organigrama de la Universidad La Salle

\section{Organograma - Universidade La Salle}

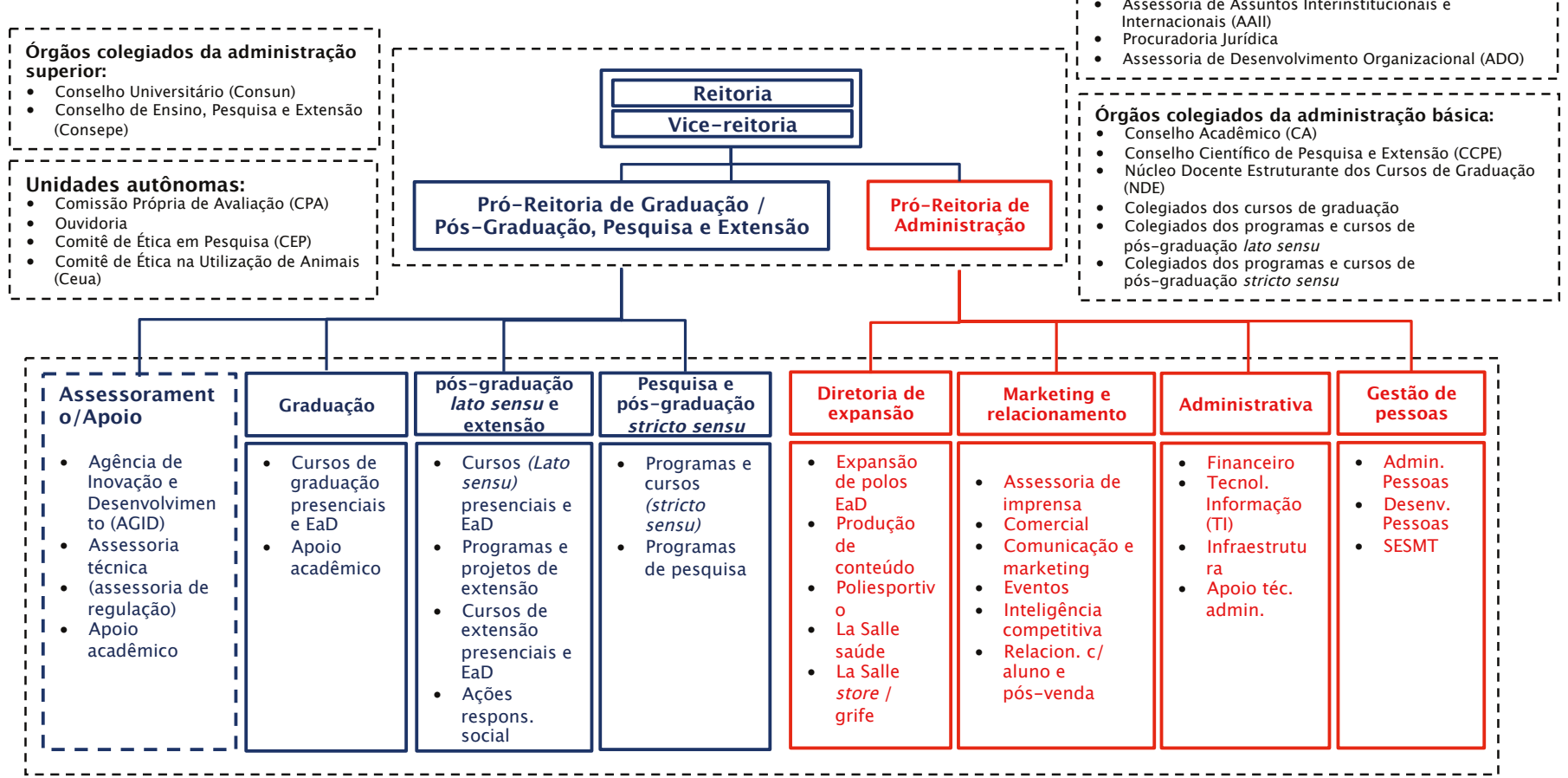

Fuente: Unilasalle (2019, p. 51). 
La Rectoría adoptó, en 2006, la herramienta estratégica Balanced Scorecard (BSC), en la que a partir del mapa estratégico institucional se desarrollaron los objetivos y los indicadores. Con sus respectivas metas, es posible acompañar el desarrollo de la institución según las siguientes perspectivas: clientes, financiero, procesos internos y aprendizaje y crecimiento. En este grupo de monitoreo están involucrados los principales liderazgos de la institución: rector, vicerrector, pro-rectores, directores, asesores, liderazgos de procesos y de cursos académicos. Los principales indicadores son acompañados, evaluados y discutidos con objeto de garantizar el alcance de las metas y el crecimiento institucional (Unilasalle, 2017). El liderazgo también se hace presente en acciones institucionales que llevan a la implementación de proyectos de naturaleza administrativa y académica. La Rectoría, las pro-rectorías, los directores y demás liderazgos interactúan con la sociedad, participando activamente en entidades de los niveles municipal, regional, de los estados, nacional y mundial, de diferentes segmentos. Los representantes comunican los objetivos estratégicos de la Unilasalle, identificando y acercando aliados a las metas institucionales (Unilasalle, 2015).

En lo que atañe a la comunicación y divulgación de las informaciones y decisiones institucionales, fue creado un modelo estandarizado de control sobre las informaciones para todos los colaboradores, identificado como Unilasalle Comparte (Unilasalle, 2015). Su objetivo es organizar y sistematizar la difusión de informaciones a los colaboradores por los liderazgos a sus equipos.

Desde 2010, y con revisión anual, la evaluación y el desarrollo de los líderes ocurre por medio del programa Gestión por Competencias (Unilasalle, 2019). El instrumento de evaluación de competencias y habilidades utilizado por la Institución trata de los factores y competencias requeridas para el desarrollo de líderes y demás miembros de la fuerza de trabajo (Fossatti, Souza y Jung, 2017). La metodología utilizada para realizar las evaluaciones es de $180^{\circ}$, en la cual el colaborador es evaluado por tres colaboradores (liderazgo superior, pares y el mismo colaborador), además de un representante del sector Gestión de Personas (que sirve como mediador y busca el consenso de la comisión). El colaborador tiene asimismo la oportunidad de realizar su autoevaluación a partir del perfil de cargo. Con base en los resultados, el sector de Gestión de Personas hace un análisis junto al liderazgo del colaborador evaluado para estructurar un feedback. Estos deben construir, juntamente como colaborador, un plan de desarrollo individual, que sirve como una de las formas de levantamiento de necesidades de entrenamiento y desarrollo individual (Unilasalle, 2015). 
Las necesidades de capacitación y desarrollo en la Unilasalle, en cuanto al claustro docente y el equipo de técnicos administrativos, se identifica mediante el Plan de Carrera Docente y el Plan de Carrera Técnico-Administrativo (Unilasalle, 2019), que le permite al colaborador visualizar las categorías que la Institución ofrece y sus requisitos. La capacitación y el desarrollo se tienen en cuenta en el Programa de Formación Continua (Unilasalle, 2019), y se emprenden a lo largo del año. El acompañamiento del desempeño de estos colaboradores se realiza por los respectivos liderazgos y, cuando es necesario, con subsidios del sector Gestión de Personas y Pastoral Universitaria. Esto se lleva a cabo con la intención de crear espacios únicos de integración, convivencia y cultivo de la fraternidad, además de que busca garantizar más calidad a las relaciones interpersonales, repensar las relaciones y reflexionar colectivamente sobre el quehacer cotidiano.

La metodología es participativa, lo cual privilegia la construcción colectiva, fundamentada en el Plan de Formación Lasalista (Unilasalle, 2015), en el Programa Calidad de Vida en el Trabajo - Salud del Trabajador (Unilasalle, 2015) y en el Programa de Gestión por Competencias (Unilasalle, 2019). Los encuentros se dividen en cuatro etapas y se dinamizan con el fin de: a) acercar a las personas y los grupos de trabajo; b) verificar y dialogar sobre cómo andan las cosas "sencillas" en la Institución (recepción, respeto, cordialidad); c) valorar la misión de cada uno de los colaboradores en la Institución, animándolos, con cariño, a asumir la misión de educadores lasalianos; d) profundizar la comprensión de la identidad y la espiritualidad lasalianas, desde la misión, la visión y los principios institucionales.

Los principales programas de desarrollo de liderazgos innovadores están descritos en la tabla 2 , así como la adecuación de espacios de creación compartida, como una forma de estimular gestores, colaboradores y demás partes interesadas a vivenciar y crear prácticas en ambientes de innovación. 
Tabla 2. Programa de desarrollo de liderazgos innovadores de la Unilasalle

\begin{tabular}{|c|c|}
\hline Programa & Objetivos \\
\hline $\begin{array}{l}\text { Curso de formación de } \\
\text { liderazgos lasalianos en Roma }\end{array}$ & $\begin{array}{l}\text { Formar líderes dentro de la filosofía lasaliana, con visión } \\
\text { de la Red La Salle Internacional, compartiendo prácticas } \\
\text { y modelos de gestión, y asimismo desarrollando trabajos } \\
\text { conjuntos en red - International Association Lassalian } \\
\text { University (IALU) (2020). }\end{array}$ \\
\hline $\begin{array}{l}\text { Curso de formación lasaliana por } \\
\text { la Universidad Corporativa La } \\
\text { Salle en modalidad Educación a } \\
\text { Distancia (EaD) }\end{array}$ & $\begin{array}{l}\text { Formar todos colaboradores y líderes con el objetivo de } \\
\text { ajustarlos a los principios filosóficos lasalianos. }\end{array}$ \\
\hline $\begin{array}{l}\text { Programa de Posgrado en } \\
\text { Gestión de Instituciones de } \\
\text { Enseñanza Superior por el } \\
\text { Consorcio de las Instituciones } \\
\text { de Enseñanza Superior (IES) } \\
\text { Comunitarias (Comung) }\end{array}$ & $\begin{array}{l}\text { Formar gestores académicos dentro de los retos que } \\
\text { necesitan las IEs Comunitarias. }\end{array}$ \\
\hline $\begin{array}{l}\text { Formación del Núcleo de } \\
\text { Inteligencia Política (NIP) }\end{array}$ & $\begin{array}{l}\text { Capacitar y desarrollar un grupo específico de liderazgos } \\
\text { académicos y no académicos que sea valorado y preparado } \\
\text { a prospectar relaciones político-institucionales por área del } \\
\text { conocimiento, que podrán constituir en la Universidad La } \\
\text { Salle los principales gestores de las áreas, centros, escuelas } \\
\text { o unidades de la organización. }\end{array}$ \\
\hline $\begin{array}{l}\text { Coaching y Mentoring para } \\
\text { gestores }\end{array}$ & $\begin{array}{l}\text { Apoyar el liderazgo para que se desarrolle en pro } \\
\text { de la gestión de cambios, con base en un plan de } \\
\text { acompañamiento que parta de los siguientes aspectos: } \\
\text { - Evaluaciones de la adecuación de las competencias } \\
\text { necesarias para actuar (NIP), a través de feedback } \\
\text { trimestral. } \\
\text { - Definición de metas para alcanzar en las reuniones de } \\
\text { feedback. } \\
\text { - Acompañar los planes de desarrollo individual (PDI), para } \\
\text { medir comprometimiento de los participantes. } \\
\text { - Plan anual de desarrollo, con conferencias y/o workshops } \\
\text { de frecuencia trimestral. } \\
\text { - Reuniones de acompañamiento de la evolución del } \\
\text { proyecto NIP, con frecuencia trimestral. }\end{array}$ \\
\hline
\end{tabular}




\begin{tabular}{|c|c|}
\hline Programa & Objetivos \\
\hline $\begin{array}{l}\text { Fomento a la formación de } \\
\text { gestores en nivel de grado y } \\
\text { posgrado ( } 50 \text { y } 80 \%)\end{array}$ & $\begin{array}{l}\text { Formación y desarrollo de liderazgos en las áreas de interés } \\
\text { de la Institución y con subsidios para cualificar la gestión. }\end{array}$ \\
\hline $\begin{array}{l}\text { Formación en metodologías } \\
\text { activas }\end{array}$ & $\begin{array}{l}\text { Desarrollar habilidades de gestores y educadores en } \\
\text { la práctica de la metodología activa, y centrada en el } \\
\text { aprendizaje. Significa una hegemonía del alumno sobre el } \\
\text { profesor, para dispensarlo en alguna medida. El alumno } \\
\text { sería un autoaprendiz. De esta manera, hay necesidad de } \\
\text { repensar las formas, los espacios y el uso de la tecnología } \\
\text { para el proceso de la enseñanza-aprendizaje. }\end{array}$ \\
\hline $\begin{array}{l}\text { Creación de espacios para } \\
\text { estimular la innovación: } \\
\text { a) FabLab, b) Room Lasallian } \\
\text { Xperience - Google for Education }\end{array}$ & $\begin{array}{l}\text { a) FabLab: el espacio del laboratorio FabLab de Unilasalle } \\
\text { tiene la proposición de promover, desde el Área de } \\
\text { Innovación y Tecnología, el desarrollo de una cultura } \\
\text { de aprendizaje a través de la práctica, de modo que se } \\
\text { proporcione un ambiente inmerso de aprendizaje. Permitir } \\
\text { a los estudiantes la realización de proyectos en los que se } \\
\text { pongan "manos a la obra”, pero, asimismo, organizando un } \\
\text { espacio transdisciplinario y abierto a la comunidad local. } \\
\text { b) Room Xperience: la alianza establecida entre la } \\
\text { Universidad La Salle, institución de educación superior } \\
\text { perteneciente a la mayor red de enseñanza de la historia } \\
\text { reciente y la mayor empresa de tecnología digital del } \\
\text { mundo: Google, fue madurada y postulada. Por tanto, más } \\
\text { allá de los saltos cualitativos pedagógicos y educacionales } \\
\text { proyectados a partir de la utilización del paquete } \\
\text { tecnológico-educacional Google for Education, se ha creado } \\
\text { una nueva unidad de resultado a la Universidad La Salle: } \\
\text { un espacio/tiempo en el que resulta posible implementar } \\
\text { prácticas educacionales y formativas -formales e } \\
\text { informales- mediante un nuevo mindset proveniente de } \\
\text { una construcción epistemológica fundada en dos lentes: } \\
\text { la Lasallista Lense y la Google For Education Lense. De esa } \\
\text { construcción emerge la Lasallian Xperience Room (LXR) - } \\
\text { Google for Education: lugar que traduce la ambientación } \\
\text { sostenida por los principios institucionales de Google y } \\
\text { Unilasalle. }\end{array}$ \\
\hline
\end{tabular}




\begin{tabular}{|l|l|}
\hline \multicolumn{1}{|c|}{ Programa } & \multicolumn{1}{c|}{ objetivos } \\
\hline $\begin{array}{l}\text { Formación en Interpretación } \\
\text { de los Criterios de Excelencia y } \\
\text { Evaluador del Premio Calidad }\end{array}$ & $\begin{array}{l}\text { Desarrollar gestores con capacidad de administrar los } \\
\text { procesos desde el modelo de excelencia de la gestión (MEG), } \\
\text { estableciendo sistemas de control de planes y prácticas de } \\
\text { trabajo orientados a la mejora continua de los procesos } \\
\text { institucionales. }\end{array}$ \\
\hline $\begin{array}{l}\text { Grupo de investigación: } \\
\text { Gestión Educacional en } \\
\text { diferentes contextos. Alcance } \\
\text { local, regional, nacional e } \\
\text { internacional }\end{array}$ & $\begin{array}{l}\text { Involucrar y desarrollar investigadores en la gestión de } \\
\text { sistemas de enseñanza y/o de instituciones educativas, en } \\
\text { el contexto de las políticas públicas sociales, considerando } \\
\text { las diferentes concepciones teóricas de Estado y de } \\
\text { ciudadanía. Enfoca los mecanismos de producción de las } \\
\text { desigualdades sociales y educacionales, confrontándolas } \\
\text { con las políticas públicas sociales. Desarrolla } \\
\text { Liderazgos }\end{array}$ \\
\hline $\begin{array}{l}\text { investigaciones para subsidiar diagnósticos, análisis, } \\
\text { proposiciones, programas y proyectos en las áreas de las } \\
\text { políticas públicas. }\end{array}$ \\
\hline $\begin{array}{l}\text { Programa definido por resolución de rectoría y orientado a } \\
\text { los potenciales liderazgos de la Institución, de acuerdo con } \\
\text { el plan de carrera y desarrollo de personas. }\end{array}$ \\
\hline
\end{tabular}

Fuentes: Unilasalle $(2015 ; 2019)$.

Como fue posible observar, la Unilasalle invierte fuertemente en la formación de nuevos liderazgos innovadores, caminando a pasos largos hacia su misión: "Ser, en 2025, una universidad reconocida por la excelencia académica y por la internacionalización” (Unilasalle, 2019, p. 22, traducción propia). Este elemento lo trata Unilasalle en su PDI, en especial en las políticas de personal, en líneas generales, considerando las orientaciones relativas a los procesos de selección y contratación, de capacitación y desarrollo, de progresión en la carrera y de participación en órganos colegiados de la Universidad.

Su cuadro de personal está formado por los integrantes del claustro docente, del equipo técnico administrativo y de los tutores. La gestión de personal implementa el proceso integrado y ajustado a los objetivos institucionales, teniendo como referencia la identidad lasaliana (Provincia La Salle Brasil-Chile, 2014), el respeto a la conducta ética y a los principios y valores profesados y la búsqueda de la excelencia.

De la misma forma, ofrece condiciones de trabajo, bienestar y desarrollo adecuadas para garantizar el proceso de la formación continua destinado a docentes, 
colaboradores y liderazgos (Unilasalle, 2019). En este sentido, se verifica que la Institución está atenta a su proceso de preparación y sucesión de liderazgos, identificando y dando oportunidades a que nuevos y futuros gestores institucionales tengan claridad de sus potencialidades. Esta organización puede ser evidenciada a través del plan de carrera docente, técnico-administrativo y demás políticas institucionales de gestión de personas (Unilasalle, 2019).

\section{Elección estratégica de los participantes}

Desde comienzos de la década de 2010, y con revisión anual, la Unilasalle realiza la evaluación y el desarrollo de los líderes por medio del programa Gestión por Competencias. El instrumento de evaluación de competencias y habilidades utilizado por la Institución trata sobre los factores y las competencias requeridos para el desarrollo de líderes y demás colaboradores. Atenta a nuevas metodologías, ha implementado más recientemente la metodología de análisis de perfil comportamental, que tiene cuatro factores comportamentales: dominancia, influencia, estabilidad y conformidad (DISC) (Unilasalle, 2015).

Esta metodología permite el mapeo de los liderazgos, lo que permite diseñar los planes de desarrollo personal/profesional. De esta forma, ha llevado a identificar posibles potenciales por medio del Programa de Desarrollo de Liderazgos, ampliando en grupo e involucrando también cargos de las diversas áreas de la Institución. Las competencias deseadas para los líderes de la Institución han posibilitado el mapeo de perfiles comportamentales, como: interacción social, comunicativo, persuasivo, creación de ambiente agradable, inspirador de confianza (Unilasalle, 2019).

De esta manera, se percibe cuanto la Institución ha dedicado efectivos esfuerzos, apoyada en las mejores prácticas de gestión, en sentido de darle vía a la preparación de sus equipos, en especial los liderazgos, de forma clara y de conocimiento de todos, aplicando la gestión. Dicha formación se estructura en planes y proyectos de formación continua, que involucran procesos y tecnologías actuales, adecuados para la perpetuación de la Institución de forma sostenible y ajustada a los principios fundacionales (Fossatti, Souza y Jung, 2017).

\section{Gestión por competencias}

En esta categoría, la Institución describe en su PDi la forma como trata la implantación de la gestión por competencias (Villarroel y Bruna, 2017), soporte necesario para los gestores y demás colaboradores, en sus respectivos procesos de desarrollo personal y profesional. Ese modelo de gestión ofrece las orientaciones en cuanto a 
conocimientos y habilidades sobre qué se espera de cada funcionario o gestor, y se apoya en la práctica de evaluación y desarrollo profesional. De este modo, se analizan con mayor relieve los puntos fuertes y positivos, y para los aspectos que necesitan ser desarrollados. Además, existe el aporte de elementos y subsidios para los procesos de selección, evaluación y gestión de personas (Unilasalle, 2019).

Con estos elementos, se evidencia la claridad que tiene Unilasalle sobre sus procesos de gestión de personas, desplegándolos de forma ajustada a su estructura de cargos, desde el diseño del perfil deseado en los distintos niveles, por medio del plan de carrera, tanto para docentes como para técnicos administrativos (Neidhart y Lamb, 2016).

\section{Autodesarrollo personalizado}

La propuesta educativa lasaliana (Provincia La Salle Brasil-Chile, 2014) comprende un perfil de educador no exclusivo al claustro docente, ya que comprende, en sentido lato, que toda persona educa por medio de sus conocimientos, actitudes y valores. Así, involucra no solo los docentes en sus responsabilidades con la enseñanza, sea en aulas presenciales o en los ambientes virtuales a distancia, puesto que incluye asimismo jefes de carrera, tutores y demás integrantes del equipo técnico-administrativo. Todos ellos participan en el concepto de actores de la educación lasaliana como personas y profesionales (Unilasalle, 2019).

La Universidad entiende que, al ofrecer condiciones para que el educando pueda aprender a conocer, a hacer, a convivir y a ser (Delors, 1999) está manifestando su expectativa con relación al "docente educador" en términos de contribución, participación y entrega en el proceso de enseñanza-aprendizaje. Específicamente sobre el ejercicio docente, Unilasalle espera que, orientado por un plan de enseñanza, el docente y los demás equipos involucrados en el proceso de enseñanza-aprendizaje contribuyan a la formación de estudiantes para la vida y para el mundo del trabajo.

Dicho perfil busca atender a las siguientes premisas: 1) comprometido con la actualidad del conocimiento y con el desarrollo de todas las dimensiones que evidencian la formación integral del educando; 2) efectiva mediación pedagógica y cultural; 3) atento a las necesidades de los educandos y que contribuya para hacer placentero el hecho de comprender, descubrir, construir y reconstruir el conocimiento; 4) que facilite el proceso de aprendizaje a partir de la identificación de las potencialidades y dificultades individuales, y promueva situaciones de estímulo al desarrollo de proyectos comunes para evidenciar posibilidades de trabajo en equipo; 5) que estimule el desarrollo de la imaginación, de la creatividad, del espíritu emprendedor, de la 
innovación, para promover el crecimiento personal del educando; 6) que incentive y sensibilice el educando a expandir el conocimiento hacia más allá de los límites propuestos en los planes de enseñanza, y a aplicarlo en situaciones reales; 7) que desarrolle la práctica de la observación y del registro de situaciones que evidencian el ritmo de aprendizaje de cada educando y la comprensión de las entregas posibles; 8) que reflexione sobre la práctica docente individual y señale necesidades para "autodesarrollo" (Unilasalle, 2019).

Para que estas características se desarrollen como proceso de formación continua, Unilasalle sobrepasa el cuidado con sus colaboradores y liderazgos, teniendo en cuenta la claridad con que expresa sus propósitos en los documentos, y en especial en las prácticas, orientadas a la formación de un perfil integral de egresados.

\section{Ajuste de la práctica de desarrollo del liderazgo en la Unilasalle basado en sus principios y valores}

Esta categoría trata del ajuste entre el planeamiento y la ejecución de las prácticas de desarrollo de liderazgos, orientados por el propósito institucional, con acciones efectivas percibidas por la comunidad académica y la sociedad (Maney, King y Kiely, 2017). Una de las evidencias sobre esta temática trata de la identificación y el análisis de las necesidades y expectativas de los clientes. Ello se da por los resultados de acciones relacionadas con el mercado, que es compromiso del Núcleo de Inteligencia Competitiva, bajo la responsabilidad del sector de Marketing de la Institución.

Dichas prácticas se evidencian en las políticas de gestión de personas y de identificación y análisis de las necesidades y expectativas de los clientes (Unilasalle, 2015). Se muestran asimismo en las políticas de gestión de comunicación y de relaciones, que consideran dos ejes: la comunicación con la sociedad y la comunicación con la comunidad académica (Etzkowitz y Zhou, 2017). Estos ejes se dan por medio de procesos, de distintos canales y medios de comunicación utilizados, de los recursos adoptados para divulgación de los compromisos de la Institución junto a sus públicos. Para todos ellos se destaca la responsabilidad social de la universidad (Andrade, 2016), así como la responsabilidad social personal. Esas prácticas buscan también demostrar la transparencia de la Universidad junto a la comunidad, y calificar la interlocución con los educandos y usuarios de los servicios educacionales ofrecidos, e igualmente con los colaboradores (Unilasalle, 2019).

El documento orientador para el funcionamiento de la IES es el PDI, desarrollado en consonancia con los instrumentos reguladores del sistema de enseñanza superior. Tal documento tiene en cuenta los factores que orientan las políticas, los programas, 
los proyectos y las respectivas acciones en consonancia con su misión, visión y principios. En esta cultura institucional, los liderazgos de la IEs son invitados a desarrollar sus itinerarios formativos.

Los resultados presentados, de acuerdo con el periodo analizado, desde comienzos de la década de 2010 hasta 2019, confirman que la IEs en estudio ha atendido los supuestos básicos de la gestión y el liderazgo, evidenciados en los documentos y políticas institucionales y en sus prácticas.

\section{Consideraciones finales}

En la actualidad es necesario el desarrollo de personas en sus competencias técnicas, humanas y transversales, con enfoque en gestión innovadora, estratégica y por resultados. Las actitudes del liderazgo transformador pasan necesariamente por un minucioso plan de trabajo y organización, fundamentales para el desarrollo de habilidades y competencias alineadas con lo esperado para el contexto del siglo xxI.

En este artículo se abordó el tema de la excelencia en la gestión del desarrollo de liderazgos en la Unilasalle. Se pudo mostrar aquí que liderar es mucho más que administrar. Es un ejercicio que involucra personas y colectividades, valores personales e institucionales, desarrollo de competencias y habilidades ajustadas con la cultura institucional y con procesos de innovación y espíritu emprendedor. El objetivo del escrito se ha centrado en mostrar las buenas prácticas del proceso de desarrollo de liderazgos organizacionales en Unilasalle. En ella se han evidenciado decisiones que involucran discursos y prácticas de liderazgo ajustados a la cultura institucional.

El desarrollo de liderazgos innovadores, en armonía con la gestión humanizada en el ambiente laboral de la IES, permite garantizar los comportamientos y resultados de los procesos internos, con vistas a la excelencia de la gestión. Dicho estilo de liderazgo lleva a procesos deseables en la atención y el aporte de un servicio efectivo de calidad a los públicos interno y externo, con diferenciales competitivos de una IEs que tiene por base los supuestos de su identidad institucional.

La Unilasalle se ha mostrado proactiva en el acompañamiento y desarrollo de sus liderazgos, de forma sistemática, involucrando hard y softs skills, inteligencia racional, emocional y adaptativa. Sus resultados registran un posicionamiento estratégico institucional por la formación de líderes de manera sistemática y personalizada. Así mismo, evidencian la necesidad del desarrollo de personas en sus competencias 
técnicas, humanas y transversales, con enfoque en gestión innovadora, estratégica y por resultados.

El surgimiento de liderazgos gestores e innovadores en la universidad en estudio emerge de una práctica basada en el trabajo en equipo, en la pedagogía de proyectos y con enfoque en indicadores, metas y resultados. Ese nuevo modo de percibir y de ser se muestra como consecuencia de un nuevo momento institucional, el cual apuesta por la promoción del desarrollo de personas, el incremento del profesionalismo, el compromiso y la satisfacción con el trabajo que se viene realizando. Por tanto, los resultados sugieren la necesidad de un proceso continuo de formación y desarrollo de líderes.

El avance del conocimiento está, entre otros, en la premisa de que formar líderes educacionales para el siglo xxi es un proceso complejo y constante, con innúmeras variables que intervienen en el ajuste del perfil deseado. Las limitaciones del estudio, circunscritas a la Unilasalle, pueden fomentar la continuidad de la investigación en otros escenarios institucionales considerando que la formación de líderes para el contexto de la innovación es fenómeno global e irreversible.

Un estudio profundo sobre los efectos del liderazgo lasaliano innovador todavía se hace necesario, teniendo en cuenta la percepción de los colaboradores y la comunidad académica. Asociar elementos que complementan la Pesquisa de Clima Organizacional (PCO) y el Programa de Avaliação Institucional (Proavi), ambos programas ya sistematizados en la Institución, será de fundamental importancia. Esta asociación tiene como objetivo diagnosticar e implementar lineamientos que apoyan la propuesta de Desenvolvimento de Lideranças Inovadoras, un plan de formación y desarrollo de personas, que está en continuo perfeccionamiento en la IEs, la cual considera aspectos técnicos y aspectos comportamentales, en conexión con este estilo de liderazgo exigido para el siglo xxI.

\section{Sobre los autores}

Renaldo Vieira de Souza. Doctor en Educación por la Universidad La Salle (2018). Máster en Ingeniería: Energía Ambiente y Materiales por la Universidad Luterana de Brasil (2005). MBA en Gestión de Instituciones de Enseñanza Superior por la Universidad de Caxias do Sul (2013). Licenciado en Administración por la Universidad Luterana de Brasil (2001). Se desempeña como jefe de gabinete, gestor de proyectos, planeamiento y desarrollo institucional. Profesor de pregrado y posgrado e investigador en la Universidad La Salle. Fue director de Administración (2015-2018), con lo cual contribuyó al proceso de preparación y acreditación por el MEC de la Universidad La Salle en 2016. Es 
profesor invitado en cursos de posgrado en la Pontificia Universidad Católica de Rio Grande do Sul. Integrante del Grupo de Investigación: Gestión Educacional en los Diferentes Contextos, vinculado al Programa de Posgrado en Educación de la Universidad La Salle.

Hildegard Susana Jung. Doctora en Educación por la Universidad La Salle (2018). Máster en Educación por la URI (2015). Especialización en Docencia en la Educación Superior (2020) y en Psicopedagogía Institucional (2009). Licenciada en Normal Superior por la Facultad de Tecnología y Ciencias (2007). Profesora y coordinadora del curso de Pedagogía e investigadora permanente del Programa de Posgrado en Educación de Unilasalle, en la línea de Formación docente, teorías y prácticas educativas. Tiene experiencia en educación básica, técnica y de idiomas, con énfasis en la formación de profesores. Segunda líder del grupo de investigación Gestión Educativa en Diferentes Contextos, vinculado al Programa de Posgrado en Educación de la Universidad La Salle.

Paulo Fossatti. Posdoctorado en Ciencias de la Educación. Investigador asociado de la Universidad de Algarve (2011). Doctorado en Educación (aprobado con honores) por la Pontificia Universidad Católica de Rio Grande do Sul (2009). Magíster en Psicología Social e Institucional de la Universidad Federal de Rio Grande do Sul (2002). Licenciado en Filosofía (1993) y en Psicología (1997) por la Universidad de Vale do Rio dos Sinos. Especialista en Administración Escolar por Unilasalle (1999). Profesor e investigador del Programa de Posgrado en Educación de la Universidad La Salle y rector de la Universidad La Salle. Tiene una amplia experiencia en la gestión de instituciones de la Red La Salle en Brasil. Presidente de la Asociación Nacional de Educación Católica (ANEC), en Brasil - Gestión 2014-2016 y Gestión 2018-2020. Coordinador del grupo de investigación Gestión Educativa en Diferentes Contextos, vinculado al Programa de Posgrado en Educación de la Universidad La Salle.

\section{Referencias}

Andrade, F. I. A. D. (2016). Uma proposta de responsabilidade social universitária: aproximação entre Sinaes, literatura e Unilasalle (tesis de maestría). Universidad La Salle, Canoas, Brasil.

Bardin, L. (2016). Análise de conteúdo. Lisboa: Edições.

Bergamini, C. W. (1994). Liderança: administração do sentido. São Paulo: Atlas.

Brasil. (1996). Lei n. ${ }^{\circ}$ 9.394, de 20 de dezembro de 1996. Estabelece as diretrizes e bases da educação nacional. Brasilia: Presidência da República. Recuperado de www.planalto.gov. br/ccivil_03/LEIS/19394.htm

Bresciani, L. P. y Guidelli, N. S. (2010). Inovação e qualidade de vida no trabalho: Uma visão integrada da Gestão a partir de um estudo de caso na Indústria Petroquímica do Grande ABC. Revista de Administração, 45(1), 57-69.

Bryman, A. (1992). Carisma and leadership in organizations. Londres: Sage Publications.

Cardoso, L. (2001). Gestão estratégica das organizações (5. ${ }^{\mathrm{a}}$ ed). Lisboa: Verbo. 
Castilhos, A. (1998). A dinâmica do trabalho de grupo. Río de Janeiro: Qualimark.

Cury, C. R. J. (2002). Gestão democrática da educação: exigências e desafios. Revista Brasileira de Política e Administração da Educação-Periódico científico editado pela ANPAE, 18(2), 1-12. https://doi.org/10.21573/vol18n22002.25486

Da Silva, É. F., Paulino, R. D., da Silva, F. F. y de Oliveira, C. B. A. (2019). A influência do estilo de liderança na satisfação do trabalho: estudo em uma unidade de ensino particular no município de Bananeiras-PB. Qualitas Revista Eletrônica, 20(2), 89-114. http://dx.doi. org/10.18391/req.v20i2.3985

Delors, J. (1999). Educação: um tesouro a descobrir. São Paulo: Cortez Editora.

Etzkowitz, H. y Zhou, C. (2017). Hélice tríplice: inovação e empreendedorismo universidade-indústria-governo. Estudos Avançados, 31(90), 23-48. https://doi.org/10.1590/ s0103-40142017.3190003

Fossatti, P. (2013). Perfil docente e produção de sentido. Canoas, Brasil: Editora Unilasalle.

Fossatti, P., Ganga-Contreras, F. y Jung, H. S. (2017). Reflexiones en torno a la gobernanza universitaria: una mirada desde Latinoamérica. Espacios, 38(1), 1-14.

Fossatti, P., Souza, R. V. y Contreras, F. A. G. (2015). Profesionalización de la gestión desde el programa gaucho de calidad y productividad: el caso de Unilasalle de Brasil. Revista Calidad en la Educación, 1(1), 161-186. http://dx.doi.org/10.4067/S0718-45652015000100006

Fossatti, P., Souza, R. V. y Jung, H. S. (2017). Desenvolvimento de lideranças lassalistas inovadoras: fundamentos para excelência da gestão. Anais do XVII Colóquio Internacional de Gestão Universitária, Mar del Plata, Argentina. Recuperado de https://repositorio.ufsc.br/ xmlui/handle/123456789/181076

Fossatti, P., Güths, H. y Jung, H. S. (2019). Gestão educacional: contingências da contemporaneidade. En Memorias del X Sincol: Simpósio Nacional de Educação. Frederico Westphalen: Editora da URI.

França A. C. L. y Arellano, E. B. (2002). Liderança: poder e comportamento organizacional. En Fleury, M. T. L. (Org.). As pessoas na organização (pp. 63-72). São Paulo: Editora Gente.

Gil, A. C. (2008). Métodos e técnicas de pesquisa social. São Paulo: Editora Atlas SA.

Hollander, E. P. (1978). Leadership dynamics: a practical guide to effective relationships. Nueva York: The Free Press.

International Association Lassalian University. (2020). Lasallian University Leadership Program. Saint Mary's College of California. Recuperado de www.stmarys-ca. edu/living-lasallian/campus-programs-resources/faculty-staff-opportunities/ international-lasallian-0

Lizuka, E. S. (2019). Inovação em ensino e aprendizagem: casos de cursos de Administração do Brasil. São Paulo: Empreende.

Lück, H. A. (2000). Escola participativa: o trabalho do gestor escolar. Río de Janeiro: Editora DByA.

Lück, H. A. (2012). Liderança em gestão escolar. Río de Janeiro: Editora Vozes. 
Lüdke, M. y André, M. E. D. A. (1986). Pesquisa em educação: abordagens qualitativas. São Paulo: EPU.

Maney, J. S., King, C. y Kiely, T. J. (2017). Who do you say you are: relationships and faith in catholic schools. Journal of Catholic Education, 21(1), 36-61. http://dx.doi.org/10.15365/ joce. 2101032017

Neidhart, H. y Lamb, J. (2016). Australian catholic schools today: school identity and leadership formation. Journal of Catholic Education, 19(1), 47-65.

Paro, V. H. (1997). Gestão democrática da escola pública. São Paulo: Editora Ática.

Provincia La Salle Brasil-Chile. (2014). Proposta educativa lassalista. Canoas, Brasil: Universidad La Salle.

Sarmento, D. F. Menegat, J. y Ramírez, V. (2015). Educação de qualidade e gestão pública: a construção do planejamento de uma secretaria de educação. Revista Brasileira de Política e Administração da Educação, 31(2), 313-333. https://doi.org/10.21573/vol31n22015.61730

Sherer, F. O. y Carlomagno, M. S. (2009). Gestão da inovação na prática: como aplicar conceitos e ferramentas para alavancar a inovação. Porto Alegre: Atlas.

Siqueira, J. (1995). Liderança, qualidade e competitividade. Río de Janeiro: Qualitymark.

Unilasalle. (2015). Relatório de Gestão PGQP - 2015. Canoas, Brasil: Universidad La Salle.

Unilasalle. (2017). Planejamento Estratégico: 2017-2025. Canoas, Brasil: Universidad La Salle.

Unilasalle. (2019). Plano de Desenvolvimento Institucional: 2019-2025. Canoas, Brasil: Universidad La Salle.

Villarroel, V. A. y Bruna, D. V. (2017). Competencias pedagógicas que caracterizan a un docente universitario de excelencia: un estudio de caso que incorpora la perspectiva de docentes y estudiantes. Formación Universitaria, 10(4), 75-96. http://dx.doi.org/10.4067/ S0718-50062017000400008

Zanguebuche, M. D. O., Flores, H. A., Franzoni, A. y Cândido, G. E. (2019). Estilos de liderança: a autopercepção dos acadêmicos de um curso de administração. Anais do XIX Colóqui Internacional de Gestão Universitária, Florianópolis, SC, Brasil. 